\title{
A influência dos apelos visuais em embalagens alimentícias para crianças e a decisão de compra dos pais: Um estudo sob a ótica do design da informação The influence of visual appeals on food packaging for children and the purchase decision of parents: a study from the point of view of information design
}

\author{
Amanda Rutiquewiski Gomes \& Carla Galvão Spinillo
}

\begin{abstract}
embalagem de alimentos, apelos visuais, decisão de compra, alimentação infantil, design da informação
Uma abundância de apelos visuais são empregados em embalagens de alimentos nos dias de hoje, sobretudo em produtos com baixo teor nutricional, que podem contribuir para problemas causados por uma má alimentação. Quando essas mercadorias são direcionadas a crianças, o uso de apelos pode se tornar prejudicial devido ao crescente número de casos de obesidade infantil. Desse modo, este artigo busca a influência de apelos visuais em embalagens de alimentos para crianças a partir de um estudo analítico de produtos do tipo e de um questionário com pais e responsáveis. Para isso, foram coletados 76 amostras em supermercados de Curitiba/Paraná para serem investigadas sob o âmbito do design da informação. Já o questionário foi realizadas com 13 participantes com filhos entre 2 e 12 anos. Os resultados obtidos revelaram que todas as amostras coletadas empregavam pelo menos um tipo de apelo visual, sobretudo imagens como de personagens famosos. Nas respostas dos pais, a maioria relatou que costumam comprar certos alimentos em razão do pedido de seus filhos e não levam em conta as diferentes técnicas de apelo. Por outro lado, os mesmos responderam acreditar que as crianças desejam o alimento pedido por causa desse tipo de tática. Assim, é possível inferir que os apelos visuais chamam a atenção do público infantil em produtos alimentícios e conclui-se que os pais são influenciados indiretamente por eles. Por fim, destaca-se que esses apelos ainda precisam continuar sendo explorados na área de design da informação.
\end{abstract}

food package, visual appeals, purchase decision, infant feeding, information design

\begin{abstract}
An abundance of visual appeals are employed in food packaging today, especially in products with low nutritional content, which can contribute to problems caused by poor diet. When these foods are targeted at children, the use of appeals can become harmful due to the increasing number of cases of childhood obesity. Thus, this article researches the influence of visual appeals on food packaging for children through an analytical study and also a questionnaire with parents and caregivers. For this, 76 products were collected in supermarkets in Curitiba/Paraná/Brazil to be investigated under the scope of information design. The questionnaire was carried out with 13 participants with children between 2 and 12 years old. The results obtained revealed that there was at least one type of visual appeal in all collected samples, especially images such as famous characters. In the responses of the parents, most reported that they usually buy certain foods because their children request it and do not take into account the different techniques of appeal. On the other hand, the participants responded that they believe that children crave the requested food because of this type of tactic. Thus, it is possible to infer that visual appeals attract children's attention to food products and it is concluded that they influence parents indirectly. Finally, it should be noted that these appeals still need to be explored in the area of information design.
\end{abstract}

\section{Introdução}

Atualmente, para chamar a atenção do público desejado, os apelos visuais de marketing são abundantes em alimentos embalados (Cairns, Angus, Hastings \& Caraher, 2013), como mascotes, i.e., animais, pessoas ou objetos ilustrados que representam uma marca ou produto. Em relação às crianças, Cairns, Angus e Hastings (2009) indicam que estes apelos visam transmitir diversão, fantasia, inovação e também o sabor da mercadoria.

Entretanto, a ilusão de valor que o marketing provoca pode ser prejudicial, já que o uso de apelos visuais é mais frequente em alimentos que podem causar dano a saúde (Lapierre, Vaala

Anais do 9 CIDI e 9 CONGIC

Luciane Maria Fadel, Carla Spinillo, Anderson Horta, Cristina Portugal (orgs.)

Sociedade Brasileira de Design da Informação - SBDI

Belo Horizonte | Brasil | 2019

ISBN 978-85-212-1728-2
Proceedings of the 9th CIDI and 9th CONGIC

Luciane Maria Fadel, Carla Spinillo, Anderson Horta, Cristina Portugal (orgs.)

Sociedade Brasileira de Design da Informação - SBDI

Belo Horizonte | Brazil | 2019

ISBN 978-85-212-1728-2 
\& Linebarger, 2011), como produtos com alto teor de açúcar, sódio e/ou gordura. Estes são consumidos em excesso pela população brasileira, ao contrário da ingestão de frutas, legumes e verduras (Brasil, 2011). Isto é particularmente preocupante na alimentação de crianças no país, pois o consumo de alimentos com baixo teor nutricional pode levar à obesidade. Em 2016, segundo a Organização Mundial da Saúde - OMS (2018), existiam mais de 380 milhões de crianças e adolescentes acima do peso ou obesos no mundo. Em razão disso, a obesidade infantil é um dos maiores problemas de saúde do século 21 para a OMS (2017).

Considerando o contexto acima, este artigo discute o uso de apelos visuais nas embalagens de alimentos para crianças sob o viés do design da informação a partir de um estudo analítico e de um questionário sobre a influência dos apelos na decisão de compra dos pais.

\section{Alimentação infantil}

Uma alimentação saudável é aquela que atende equilibradamente as questões biológicas de cada corpo e que impede o consumo em excesso de gordura, fato que pode aumentar o risco de desenvolvimento prematuro de transtornos de saúde (Brasil, 2014; OMS, 2017). Para auxiliar nas escolhas saudáveis, existem diversos documentos oficiais como o Modelo de Perfil Nutricional da Organização Pan-Americana da Saúde - OPAS (2016), que assiste na prevenção da obesidade em crianças com táticas que regulam e alertam sobre o marketing, a merenda escolar e o uso de rótulos nutricionais. Em nosso país, o Guia Alimentar para a População Brasileira (Brasil, 2014) também traz indicações sobre o consumo de alimentos por crianças e o planejamento de refeições para o público infantil.

Entretanto, é possível afirmar que os ensinamentos e as advertências sobre alimentação podem ter pouco efeito se o marketing é capaz de levar a uma interpretação equivocada dos dados da rotulagem. Assim, não só as informações nutricionais tem um papel relevante nas embalagens de alimentos, mas também os apelos encontrados nesses produtos. É por isso que os apelos de marketing em alimentos embalados podem ser prejudiciais e merecem atenção quando se trata do público infantil (OMS, 2017), ainda mais quando estes são ultraprocessados, ou seja, alimentos que envolvem várias etapas de produção e possuem muitos ingredientes, incluindo aqueles exclusivos da indústria (Brasil, 2014).

\section{Uso de apelos de marketing em embalagens de alimentos para o público infantil}

Os apelos visuais empregados em alimentos e bebidas para crianças podem ser apresentados de diversas formas. Estes podem ser personagens, mascotes, celebridades, brindes, promoções, entre outros (Giménez, Saldamando, Curutchet \& Ares, 2017). Ainda, encontramse alegações nutricionais e de saúde sobre a existência ou não de uma determinada substância (e.g., sem gordura trans) e que referenciam ao gosto ou à energia proporcionada (Giménez et al., 2017)

O emprego de apelos em embalagens de alimentos infantis já é um tema explorado internacionalmente. No Brasil, pesquisadores observaram, em alimentos comumente consumidos pelo público infantil, que $75 \%$ deles apresentam algum apelo direcionado especificamente a crianças (Ferreira, Silva, Moraes \& Tancredi, 2015). Os números são ainda maiores no exterior: $76 \%$ produtos alimentícios infantis contém algum personagem no Uruguai (Giménez et al., 2017) e, na Guatemala, 92,5\% (Chacon, Letona \& Barnoya, 2013).

Já sobre uma das possíveis influencias dos apelos visuais, Lapierre et al.(2011) observaram que crianças americanas gostam mais do gosto de certos alimentos quando estes apresentam personagens animados famosos em suas embalagens. Além disso, os autores ainda relataram que quando o nome da mercadoria sugere algo saudável, esta também é tida como a mais saborosa pelas crianças.

Além da visão do marketing em relação aos apelos visuais nas embalagens de alimentos, estes também podem ser caracterizados pelo design da informação, como linguagem gráfica nos modos de simbolização pictórico, verbal e esquemático (Twyman, 1985). Com base nessa divisão, os apelos visuais podem ser segmentados como: 
Gomes, A. R. \& Spinillo, C. G. | A influência dos apelos visuais em embalagens alimentícias para crianças e a decisão de compra dos pais: um estudo sob a ótica do design da informação

- Pictórico: personagem licenciado de TV, filme ou HQ; mascotes; celebridades; imagens de crianças ou ilustrações em geral;

- Verbal: alegações textuais de saúde ou de nutrientes;

- Esquemático: brindes, promoções, concursos ou qualquer outra estratégia observada que se apresente como um esquema.

Outro conceito importante que pode ser relacionado aos apelos é o de retórica que, para Aristóteles (384 a.C. a 322 a. C., como citado por Mazzali, 2008), é a persuasão através da comunicação, seja ela escrita ou oral. Esta, conforme o filósofo, busca a melhor forma de convencer alguém a partir de três técnicas:

- Ethos: confiança, por exemplo, quando alguém compra um produto por crer na marca ou no fabricante;

- Pathos: emoção, relacionada aos sentimentos que levam alguém a comprar algum determinado alimento;

- Logos: verdade e raciocínio, como acreditar nas informações nutricionais e na lista de ingredientes presentes nos produtos ultraprocessados.

Com base na literatura sobre os apelos visuais de marketing e na sua relação com a linguagem gráfica e a retórica visual, a seguir é apresentado um estudo analítico e um questionário sob a perspectiva do design da informação. Estes visaram observar e compreender as embalagens de alimentos para o público infantil encontradas no Brasil.

\section{Estudo analítico e questionário sobre apelos visuais em embalagens de alimentos para crianças}

O estudo analítico objetivou observar o uso de apelos visuais na face frontal de embalagens de alimentos e bebidas para crianças. Foram coletados 76 produtos diversos em cinco supermercados da região central de Curitiba/Paraná.

Definiu-se que o protocolo (quadro 1) constaria de nome do alimento/bebida, fabricante, categoria do alimento/bebida e modo de simbolização dos apelos visuais. Foram estabelecidas 12 categorias de produtos a partir das divisões encontradas nos próprios supermercados: pães, bolos, gelatinas, biscoitos doces, salgadinhos, cereais, congelados, sucos, refrigerantes, iogurtes, leites e achocolatados. O quadro 1 demostra como o protocolo foi preenchido. Para análise dos dados, estes foram observados de forma quantitativa para definição de tendências da amostra.

Quadro 1: Protocolo do estudo analítico com exemplo de preenchimento.

\begin{tabular}{c|c|c|c|c|c}
\multirow{2}{*}{ Nome } & \multirow{2}{*}{ Fabricante } & \multirow{2}{*}{ Categoria } & \multicolumn{3}{|c}{ Apelo } \\
\cline { 4 - 6 } & & & Pictórico & Verbal & Promocional \\
\hline Snow Flakes & Nestlé & Cereal & Mascote & Texto &
\end{tabular}

Buscando entender a influência dos apelos na decisão de compra de alimentos e bebidas, foi realizado um questionário online com 13 pais de crianças entre 2 e 12 anos. Este era composto de três questões relacionadas aos hábitos de compra no supermercado, relacionando: (1) aspectos que influenciam os pais a comprarem o alimento ou a bebida para seus filhos, (2) aspectos que os pais acreditam influenciar as crianças a pedirem certo produto e (3) as informações presentes nas embalagens lidas pelos participantes antes da compra. Os resultados também foram examinados quantitativamente.

Os dados obtidos no estudo analítico e no questionário, apresentados na seção a seguir, foram então relacionadas entre si, além de também serem discutidos com a literatura apresentada anteriormente. 
Gomes, A. R. \& Spinillo, C. G. | A influência dos apelos visuais em embalagens alimentícias para crianças e a decisão de compra dos pais: um estudo sob a ótica do design da informação

\section{Síntese e discussão dos resultados}

Dos 76 produtos analisados, foram observados 34 fabricantes, sendo a Nestlé $(n=12)$ a marca com mais mercadorias. Sobre as categorias de alimento/bebida, a mais encontrada foi o achocolatado $(n=14)$. Em todas as faces frontais, foram identificados 120 apelos (gráfico 1), sobretudo pictóricos $(n=71)$. Destes, os mascotes $(n=27)$ são os mais utilizados, seguido dos personagens $(n=24)$, principalmente de filmes $(n=12)$. Já quando os apelos são empregados de forma conjunta (gráfico 2), a combinação mais usada é de pictóricos e textuais $(n=35)$. A figura 1 ilustra algumas destas tendências em embalagens de alimentos e bebidas para crianças. A imagem da esquerda apresenta um apelo visual pictórico da televisão (desenho animado, Bob Esponja) juntamente com um apelo verbal de que o produto é uma fonte de vitaminas. Já na outra embalagem é empregado um mascote, o Chester Cheetos.

Gráfico 1: Incidência dos apelos nos três modos de simbolização.

\section{TOTAL: 120}

\section{pictórico: 71}

mascote: 27

\section{ilustração: 20}

\section{personagem: 24}

história em quadrinhos: 5

filme: 12

televisão: 7

\begin{tabular}{|c|}
\hline verbal: $\mathbf{4 2}$ \\
\hline texto: 42 \\
\hline
\end{tabular}

esquemático: 7

concurso: 5

brinde: 2

Gráfico 2: Uso conjunto dos apelos nos modos de simbolização.

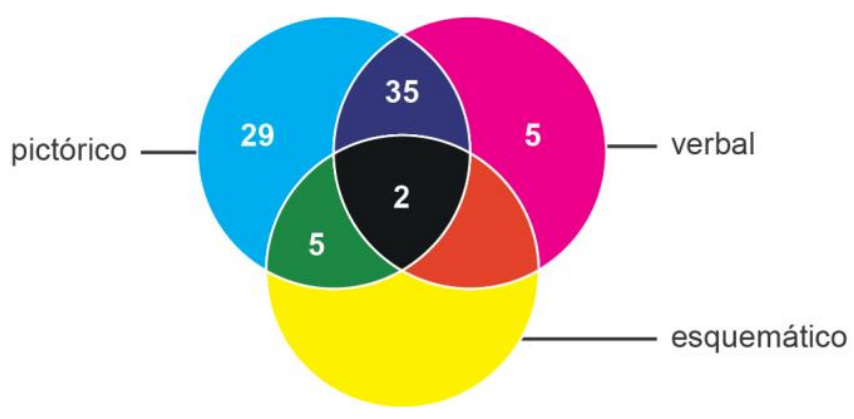


Figura 1: Exemplo do uso de apelos em embalagens de alimentos e bebidas. Fonte (esquerda): Elegê. Recuperado em 4 de julho, 2019 em http://elege.com.br/produtos/leite-fermentado-bob-esponja-tradicional-80g/; Fonte (direita):

PepsiCo. Recuperado em 4 de julho, 2019 em https://www.lojapepsico.com.br/300032051_cheetos_onda_57g/p
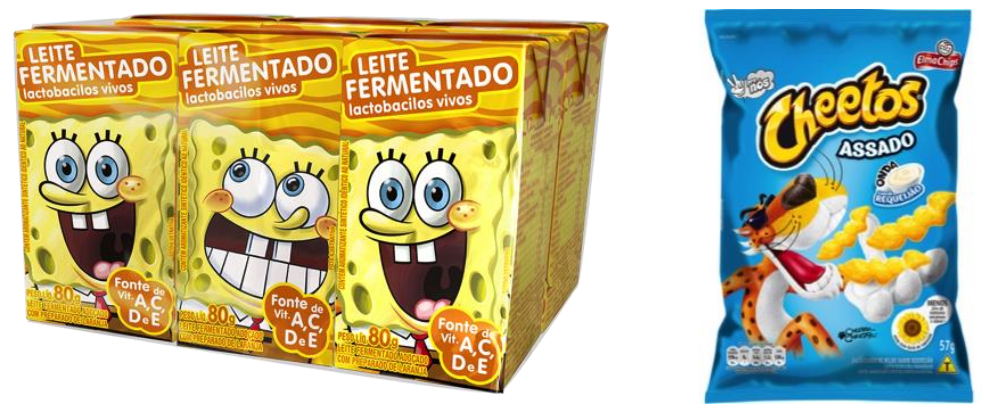

Estes dados mostraram que os fabricantes utilizam diversos tipos de apelos de marketing para chamar a atenção das crianças, conforme revelaram os estudos de Chacon et al. (2013), Ferreira et al. (2015) e Giménez et al. (2017). Pela quantidade de apelos pictóricos de mascote e personagens, é possível inferir que figuras conhecidas pelo público infantil são preferíveis a qualquer outro tipo de apelo, assim como observado por Lapierre et al. (2011). Alguns exemplos encontrados na amostra foram Bob Esponja e Chester Cheetos, como na figura 1, além de Galinha Pintadinha, Turma da Mônica e Minions. O número de alimentos e bebidas relatados nesta pesquisa que apresentaram um desses dois tipos de apelo pictórico (52 de 76) chega a $68 \%$, parecido com os $63 \%$ encontrados por Ferreira et al. (2015). A utilização desses apelos visuais ainda está mais baixa do que identificado em outros países (Chacon et al., 2013; Giménez et al., 2017).

Sobre o questionário com os 13 pais (tabela 1) a respeito dos hábitos no supermercado, a maioria dos participantes respondeu atender ao pedido de seus filhos $(n=10)$ durante a compra, seguindo do reconhecimento da marca ou do fabricante $(n=9)$. Apenas um dos indivíduos relatou levar em conta os apelos de marketing existentes sobre o produto. Por outro lado, quando questionados sobre os fatores que acreditam influenciar o desejo das crianças por certo alimento, o marketing foi assinalada 8 vezes e a embalagem, 4 . Os participantes ainda afirmaram observar sobretudo a data de validade $(n=9)$ e a lista de ingredientes $(n=7)$ no momento da compra.

Tabela 1: Questionário com pais e responsáveis (múltipla escolha).

\begin{tabular}{l|c|l|c|l|c}
$\begin{array}{c}\text { Fatores que influenciam } \\
\text { os pais durante a compra }\end{array}$ & $\begin{array}{c}\text { Fatores que os pais acreditam } \\
\text { influenciar os filhos }\end{array}$ & \multicolumn{2}{|c}{$\begin{array}{c}\text { Informações que os pais } \\
\text { leem antes da compra }\end{array}$} \\
\hline Pedido dos filhos & 10 & Marketing & 8 & Data de validade & 9 \\
\hline Marca ou fabricante & 9 & Indicação dos amigos & 6 & Ingredientes & 7 \\
\hline Recomendação & 7 & Embalagem & 4 & Rótulo nutricional & 4 \\
\hline Praticidade & 6 & & & Alergênicos & 3 \\
\cline { 1 - 3 } \cline { 4 - 6 } Artigos ou notícias & 4 & & & Tabela nutricional & 2
\end{tabular}

É possível notar que os pais são motivados pelas três técnicas de retórica de Aristóteles (384 a.C. a 322 a. C., como citado por Mazzali, 2008). Em primeiro lugar, pelo pathos, por comprarem alimentos e bebidas pedidos pelos filhos (fator emocional). Depois, tanto pelo ethos, por procurarem marcas e fabricantes conhecidos e que confiam, e pelo logos, quando buscam as informações nutricionais impressas na embalagem do produto. Desse modo, percebe-se que os participantes têm uma intenção em comprar alimentos saudáveis e estão preocupados em oferecer uma alimentação mais balanceada para seus filhos. Entretanto, a influência emocional gerada pelo pedido das crianças faz com que os pais também sejam instigados indiretamente pelo marketing, seja através de apelos presentes na TV, na internet ou na própria embalagem.

Assim, verifica-se que os apelos de marketing em alimentos embalados para o público infantil são um dos fatores que influenciam os hábitos de compra no supermercado, seja dos Anais do 9 Congresso Internacional de Design da Informação | CIDI 2019 Proceedings of the 9th Information Design International Conference Anais do $9^{\circ}$ Congresso Nacional de Iniciação Científica em Design da Informação | CONGIC 2019 Proceedings of the $9^{\text {th }}$ Information Design Student Conference 
pais ou das crianças. Porém, as categorias de alimentos e bebidas vendidas nesses estabelecimentos e contempladas no estudo analítico, como os salgadinhos e os achocolatados, são em geral definidas como ultraprocessados. Por causa disso, no momento em que os participantes compram mercadorias a pedido dos filhos, estes produtos, se consumidos, podem atuar no aumento de peso das crianças.

\section{Considerações finais}

A presença de apelos visuais em embalagens de alimentos ultraprocessados ainda é uma estratégia amplamente utilizada para chamar a atenção dos consumidores, como observado por Cairns et al. (2013). Quando voltada para o público infantil, ela ganha uma ênfase especial, pois esse tipo de informação pode contribuir para a ingestão de alimentos com baixo teor nutritivo que podem causar, precocemente, sobrepeso e obesidade.

Neste contexto, este artigo mostrou que embora os pais digam não levar em conta o marketing, o pedido dos filhos é sobretudo motivado por esse fator e assim os dados do estudo ratificam que os apelos visuais em embalagens são elementos muito fortes, principalmente os pictóricos. Essa informação está em concordância com a OMS (2017), que indica que apelos podem se caracterizar como um problema quando se pensa em alimentação saudável.

É possível concluir, com o constante uso de apelos visuais de marketing encontrado nas amostras coletadas para o estudo analítico, que a decisão de compra por alimentos saudáveis para crianças pode se tornar mais difícil. Portanto, os apelos são um tema que deve ser mais discutido no ambiente acadêmico, inclusive no âmbito do design da informação, para que seu uso possa ser, futuramente, regulado por lei em nosso país.

\section{Referências}

Brasil. Ministério da Saúde. (2014). Guia alimentar para a população brasileira. Recuperado em 8 de fevereiro, 2019 de http://bvsms.saude.gov.br/bvs/publicacoes/guia_alimentar_ populacao_brasileira_2ed.pdf

Brasil. Instituto Brasileiro de Geografia e Estatística - IBGE. (2011). Pesquisa de Orçamentos Familiares 2008-2009. Recuperado em 8 de fevereiro, 2019 de https://biblioteca. ibge.gov.br/visualizacao/livros/liv50063.pdf

Cairns, G., Angus, K., \& Hastings, G. (2009). The extent, nature and effects of food promotion to children: a review of the evidence to December 2008. World Health Organization, WHO Press.

Cairns, G., Angus, K., Hastings, G., \& Caraher, M. (2013). Systematic reviews of the evidence on the nature, extent and effects of food marketing to children. A retrospective summary. Appetite, 62, 209-215.

Chacon, V., Letona, P., \& Barnoya, J. (2013). Child-oriented marketing techniques in snack food packages in Guatemala. BMC Public Health, 13(1), 967.

Ferreira, J. S. G., Silva, Y. da, Moraes, O. M. G de., \& Tancredi, R. P. (2015). Marketing de alimentos industrializados destinados ao público infantil na perspectiva da rotulagem. Vigilância Sanitária em Debate: Sociedade, Ciência \& Tecnologia, 3(2), 75-84.

Giménez, A., Saldamando, L. D., Curutchet, M. R., \& Ares, G. (2017). Package design and nutritional profile of foods targeted at children in supermarkets in Montevideo, Uruguay. Cadernos de saude publica, 33, e00032116.

Lapierre, M. A., Vaala, S. E., \& Linebarger, D. L. (2011). Influence of licensed spokescharacters and health cues on children's ratings of cereal taste. Archives of pediatrics \& adolescent medicine, 165(3), 229-234.

Mazzali, G. C. (2008). Retórica: de Aristóteles a Perelman. Revista Direitos Fundamentais \& Democracia, 4(4). 
Gomes, A. R. \& Spinillo, C. G. | A influência dos apelos visuais em embalagens alimentícias para crianças e a decisão de compra dos pais: um estudo sob a ótica do design da informação

Organização Mundial de Saúde - OMS. (2017). Facts and figures on childhood obesity. Recuperado em 2 de setembro, 2018 em http://www.who.int/end-childhoodobesity/facts/en

Organização Mundial de Saúde - OMS. (2018). Obesity and overweight. Recuperado em 2 de setembro, $2018 \mathrm{em} \mathrm{http://www.who.int/news-room/fact-sheets/detail/obesity-and-}$ overweight

Organização Pan-Americana da Saúde - OPAS. (2016). Modelo de Perfil Nutricional da Organização Pan-Americana da Saúde. Washington, DC: OPAS.

Twyman, M. (1985). Using pictorial language: A discussion of the dimensions of the problem. In Designing usable texts (pp. 245-312). Academic Press.

\section{Sobre as autoras}

Amanda Rutiquewiski Gomes, Mestranda, UFPR, Brasil <amandaruti@gmail.com> Carla Galvão Spinillo, PhD, UFPR, Brasil <cgspin@gmail.com> 